\title{
COVID-19 and Mental Health of Adolescents: A South Indian Perspective
}

Brinda Prasanna Kumar ( $\square$ brinda.skps@gmail.com )

Department of Pediatrics, Rajarajeswari Medical College and Hospital, Kambipura Karnataka, Bangalore, India. https://orcid.org/0000-0003-3649-4586

Adarsh E

RajaRajeswari Medical College and Hospital

Sahana G

RajaRajeswari Medical College and Hospital

\section{Research article}

Keywords: Mental health, COVID-19, adolescents, depression, help seeking

Posted Date: September 8th, 2020

DOI: https://doi.org/10.21203/rs.3.rs-73160/v1

License: (c) (i) This work is licensed under a Creative Commons Attribution 4.0 International License. Read Full License 


\section{Abstract}

Background: The COVID-19 outbreak and stay at home orders has had multifaceted consequences on the mental health of adolescents. Therefore, the authors aim to (1) Investigate the feelings of depression among adolescents during the COVID-19 pandemic (2) Evaluate the mental help seeking behavior and awareness among the adolescents.

Methodology: A cross-sectional survey was undertaken where adolescents in the age group of 10 to 19 years were invited to participate with a selfadministered questionnaire. A snowball sampling strategy was used, focusing on recruiting adolescents living in Bangalore, India during the outbreak of COVID-19 pandemic. The survey included the PHQ-2 scale and questions on help seeking and awareness. The questionnaires were completed in English through an online survey platform. Data collection took place over five days.

Results: The study included 300 participants with a mean age of $17.01+/-1.34$ years. PHQ-2 scale revealed a sample mean of 1.32 (SD=0.99). $34.7 \%$ felt little interest or pleasure in doing things on several days and 32.0\% felt down depressed or hopeless on more than half the days. $52.3 \%$ of the adolescents indicated that they were unlikely or were unsure about seeking help for mental health issues and $24.3 \%$ were not aware of any mental health helpline. No association between socio-demographics and PHQ-2 scale was established. Majority indicated intention to seek help from parents or siblings.

Conclusion: Adolescents constitute a vulnerable population and need careful consideration. The COVID -19 outbreak has resulted in increased depressive symptoms, anger, difficulty in relaxation and sleep disturbances. Lack of awareness of support and reluctance to use the resources has had a compounding effect on the mental health status of adolescents. Adolescents often choose to express their mental health concerns to their parents and siblings. Therefore, it is worth considering family as a potential resource of support for adolescents in this challenging time of need.

\section{Introduction}

The novel corona virus (COVID-19) was recognized first in December 2019 and since its recognition has spread rapidly throughout the globe. Not only has it brought with it health consequences but also devastating socio-psychological and economic uncertainty. Adolescence is a particularly vulnerable period and is often characterized by heightened stress ${ }^{1}$ as children adapt to the many changes such as physical maturation, brain development, social expectations and increased salience of peer interaction ${ }^{2,3}$. The COVID-19 outbreak and stay at home orders has had multifaceted consequences on the mental health of adolescents. Sudden school closures, limited physical activity and lack of peer interaction may be detrimental to the overall wellbeing of children. Prior studies have established that children who are quarantined are more likely to report high levels of depressive and stress symptoms ${ }^{4,5}$. Extended periods of home confinement may result in poor mental health outcomes and avoidance behaviors ${ }^{6}$.

Social support can buffer the negative effect of stress and depressive symptoms. It is important to understand and look for potential resilience factors that may help protect adolescents against high stress levels. Although several agencies have come forward with counseling and mental health support services for children and young adults, these services are often not well organized and directed towards specific needs of children ${ }^{7}$. In addition the implementation of these services is limited by the help seeking behavior of adolescents and awareness of their availability.

$41 \%$ of India's population is less than 18 years of age and their mental health issues cannot be neglected. Given the potential for long-lasting psychological effect there is an urgent need to determine the prevalence of psychological distress among adolescents during this pandemic and understand their mental help seeking pattern and awareness of professional help. Therefore, the authors undertake this study with the following objectives (1) Investigate the feelings of depression and anhedonia among adolescents during the COVID-19 pandemic (2) Evaluate the mental help seeking behavior and awareness among the adolescents.

\section{Methodology}

An online survey based observational study was conducted among adolescents residing in Bangalore, India during the COVID-19 pandemic outbreak. The questionnaire was developed using Google forms and was kept anonymous to ensure confidentiality and reliability of data. A snowball sampling strategy was used where the online survey was first disseminated to the contacts of the investigators on various social media platforms and they were encouraged to pass it on to their peers.

Participants in the age group of 10 to 19 years, who could understand English and with access to internet connection were invited to participate in the study. The participants self-selected into the study and were recruited upon completion of an e-consent form. The participants were provided with a letter explaining the study and were asked to provide it to their parents. Since the study involved no more than minimal risk passive parental permission was used. Data collection took place over five days from 25th April to 29th April.

The online self reported questionnaire in addition to the socio-demographic information incorporated the Patient Health Questionnaire-2 (PHQ-2) to inquire about the frequency of depressive feelings and anhedonia over the past 2 weeks. The PHQ-2 scale has been established as a reliable and valid tool to quickly screen for major depressive symptoms in adolescents. The brief nature of the instrument makes this tool a promising first step for screening adolescent depression in primary care setting ${ }^{8}$. The PHQ-2 consists of two items with total scores ranging from 0 to 6 . Based on acceptable sensitivity and specificity values a score of $\geq 3$ has been identified as an acceptable cut-off for identifying clinically significant depressive symptoms in adolescent population ${ }^{9}$. Respondents reported their symptoms using a 4-item Likert rating scale ranging from 0 (not at all) to 3 (nearly every day).

The authors included additional questions related to anger, relaxation, concentration and sleep. Mental help seeking attitudes and behavior was evaluated using the following three questions: (1) "If I feel the need I am likely to seek help." "No" response ended the questionnaire while a "Yes/Unsure" response

Page $2 / 6$ 
evoked the following questions (2) "In case of need for emotional or psychological support I am most likely to seek help from" followed by 9 variables with multiple answer choices. (3) Awareness of the presence of a helpline for counseling and emotional support was accessed by a Yes/No question.

Data was entered into Microsoft excel data sheet and was analyzed using SPSS 22 version software. Categorical data was represented in the form of Frequencies and proportions. Chi-square test was used as the test of significance for qualitative data. Continuous data was represented as mean and standard deviation. ANOVA (Analysis of Variance) was used as the test of significance to identify the mean difference between more than two groups for quantitative data. Graphical representation of data was done using MS Excel and MS word, which was also used to obtain various types of graphs such as bar diagram and pie diagram. A p-value (Probability that the result is true) of $<0.05$ was considered as statistically significant after assuming all the rules of statistical tests.

\section{Results}

\subsection{Participants}

A total of 300 individuals provided data for the survey between April 25th to 29th, 2020 and were included in the current analysis. Participants were aged $17.01+/-1.34$ years (range 10-19 years). The study group predominantly composed of females (71.3\%) and $73.0 \%$ had one or more siblings. $78.3 \%$ belonged to a nuclear family, $17.0 \%$ belonged to a joint family, $4.7 \%$ belonged to a single parent family and $81.7 \%$ of the respondents belonged to a family with three to five individuals.

\subsection{Symptoms of depression and anhedonia}

Feelings of stress and depression measured using the PHQ-2 scale revealed a sample mean of 1.32 (SD = 0.99). Table- 1 shows the response distribution to PHQ-2 scale among the study subjects. Over the past two weeks, $34.7 \%$ felt little interest or pleasure in doing things on several days and $32.0 \%$ felt down depressed or hopeless on more than half the days. $41.7 \%$ felt angered or agitated about the situation and $34.0 \%$ found it hard to relax on more than half the number of days. $32.0 \%$ faced difficulty in concentrating and 33.0\% experienced altered sleeping habits nearly every day. Our study showed no statistically significant association between socio-demographic characters and PHQ-2 scale (Table-2).

\subsection{Awareness and help seeking behavior}

$47.7 \%$ indicated that in case of need for psychological support they were likely to seek help, $13.7 \%$ indicated no intentions of seeking any help and $38.7 \%$ were unsure of their intentions to seek help. In the study $56.0 \%$ were aware of the presence of a helpline for counseling and emotional support and $24.3 \%$ were not aware of any helpline. Drawing from data represented in Figure-1, majority indicated intention to seek help from parent (57.7\%) and/or sibling (57.3\%). Mental health professional (26.0\%), friend (12.7\%) and other family members (12.3\%) were found to be the other mental help seeking sources. Doctor (9.0\%), intimate partner $(6.0 \%)$, helpline $(2.7 \%)$ and religious leaders (1.0\%) were found to be lesser sources of help.

\section{Discussion}

WHO has identified the global burden of mental health diseases among adolescents to effect $10-20 \%$ of the population and half of all mental illnesses begin by the age of 14 years. Depression has been identified as the single largest cause of disease burden among the younger population. Children with mental illnesses face major challenges related to stigma, isolation, discrimination, lack of access to healthcare and education facilities in violation of their fundamental rights ${ }^{11}$. Who has also published recommendations addressing adolescents and to help them cope with stress ${ }^{12}$.

Adolescents in the study reported high frequencies of depressed symptoms, anger, difficulty in relaxation and sleep disturbances. These findings suggest that the pandemic presents serious mental health challenges for adolescents, with potential for both short term and long term consequences. These findings suggest the urgent need to reduce the psychological distress experienced by adolescent population. The adolescents need to be screened and those with deviations from the normal need to be proactively addressed.

$52.3 \%$ of the adolescents indicated that they were unlikely or were unsure about seeking help for mental health issues and $24.3 \%$ were not aware of any mental health helpline. Studies in the past have indicated stigma associated with mental health problems to be a key deterrent to help seeking behavior ${ }^{10}$. These findings highlight the importance of undertaking sensitizing campaigns to spread awareness among adolescents. However no significant difference in gender or age indicated that male and female children of all the age groups experienced similar feelings of depression and negative emotions due to the pandemic.

Governments, mental health centers and volunteer organizations have established platforms and help lines for psychological assistance and crisis intervention, but the awareness and utilization rate of the public is not high. In India there are seven psychiatrists per one million population. Apart from a severe shortage these specialists are urban centered and carter only to $10 \%-20 \%$ of the total burden of mental health disorders ${ }^{13-14}$. Mental healthcare in India is integrated into the existing primary health system and is delivered through the National Mental Health Programme (NMHP) and District Mental Health Programme (DMHP) ${ }^{15-17}$. With strict social distancing norms in effect the access to these resources has been cut off. Therefore, as indicated by our study adolescents who are isolated at home are more likely to choose parents and siblings to alleviate their inner troubles.

The limitation of the study was that it was conducted online; therefore adolescents residing in rural areas or those without an internet connection might not have participated. Further studies with more robust sampling methods representing data from all regions are warranted. Given the strict measures implemented by the government and the time sensitivity of the COVID-19 outbreak, we adopted a snowball sampling strategy which is not based on random selection of the sample. As a result there was an oversampling of a particular network of peers and did not reflect the actual pattern of the general population.

Page $3 / 6$ 
Notwithstanding the above limitations, the findings of our study can be utilized to undertake mental health interventions to improve the psychological resilience of adolescents during the COVID-19 pandemic. Family is the closest and most evident help for children. The findings also indicate the importance of recognizing family as a potential resource of support for adolescents in this challenging time and the need to integrate mental health services not only at the school and community level but also at the level of families.

Table 1

PHQ-2 scale distribution

\begin{tabular}{|c|c|c|c|c|c|c|c|c|}
\hline \multirow[t]{2}{*}{ PHQ-2 Scale } & \multicolumn{2}{|c|}{ Not at all } & \multicolumn{2}{|c|}{ Several days } & \multicolumn{2}{|c|}{$\begin{array}{l}\text { More than half the } \\
\text { days }\end{array}$} & \multicolumn{2}{|c|}{$\begin{array}{l}\text { Nearly every } \\
\text { day }\end{array}$} \\
\hline & Count & $\%$ & Count & $\%$ & Count & $\%$ & Count & $\%$ \\
\hline How often have you felt little interest or pleasure in doing things? & 34 & 11.3 & 104 & 34.7 & 112 & 37.3 & 50 & 16.7 \\
\hline How often have you felt down, depressed or hopeless? & 74 & 24.7 & 89 & 29.7 & 96 & 32.0 & 41 & 13.7 \\
\hline \multicolumn{9}{|l|}{ Other problems faced } \\
\hline How often have you felt agitated or angered about the situation? & 48 & 16.0 & 93 & 31.0 & 125 & 41.7 & 34 & 11.3 \\
\hline How often did you find it difficult to relax? & 97 & 32.3 & 67 & 22.3 & 102 & 34.0 & 34 & 11.3 \\
\hline $\begin{array}{l}\text { How often have you had difficulty in concentrating on college/school work, } \\
\text { reading etc? }\end{array}$ & 43 & 14.3 & 84 & 28.0 & 77 & 25.7 & 96 & 32.0 \\
\hline $\begin{array}{l}\text { How often have you had trouble falling asleep, staying asleep, or sleeping too } \\
\text { much? }\end{array}$ & 61 & 20.3 & 64 & 21.3 & 76 & 25.3 & 99 & 33.0 \\
\hline
\end{tabular}

Table 2

Socio-demographic variables and PHQ-2 scale

\begin{tabular}{|c|c|c|c|c|c|c|}
\hline \multirow[t]{3}{*}{ Socio-demographic Variables } & & \multicolumn{4}{|c|}{ PHQ-2 Scale } & \multirow[t]{3}{*}{$P$ value } \\
\hline & & \multicolumn{2}{|l|}{$\geq 3$} & \multicolumn{2}{|l|}{$<3$} & \\
\hline & & Count & $\%$ & Count & $\%$ & \\
\hline \multirow[t]{4}{*}{ Age } & $<14$ years & 5 & 29.4 & 12 & 70.6 & \multirow[t]{4}{*}{0.219} \\
\hline & 15 to 16 years & 20 & 35.7 & 36 & 64.3 & \\
\hline & 17 to 18 years & 97 & 47.3 & 108 & 52.7 & \\
\hline & $>18$ years & 8 & 36.4 & 14 & 63.6 & \\
\hline \multirow[t]{2}{*}{ Gender } & Female & 98 & 45.8 & 116 & 54.2 & \multirow[t]{2}{*}{0.175} \\
\hline & Male & 32 & 37.2 & 54 & 62.8 & \\
\hline \multirow[t]{3}{*}{ Education } & Bachelors/university & 10 & 35.7 & 18 & 64.3 & \multirow[t]{3}{*}{0.145} \\
\hline & Lower secondary & 4 & 23.5 & 13 & 76.5 & \\
\hline & Upper secondary & 116 & 45.5 & 139 & 54.5 & \\
\hline \multirow[t]{3}{*}{ Type of Family } & Joint Family & 19 & 37.3 & 32 & 62.7 & \multirow[t]{3}{*}{0.576} \\
\hline & Nuclear Family & 104 & 44.3 & 131 & 55.7 & \\
\hline & Single Parent Family & 7 & 50.0 & 7 & 50.0 & \\
\hline \multirow[t]{3}{*}{ Number of individuals in the household } & Six people or more & 14 & 40.0 & 21 & 60.0 & \multirow[t]{3}{*}{0.771} \\
\hline & Three to Five people & 106 & 43.3 & 139 & 56.7 & \\
\hline & Two People & 10 & 50.0 & 10 & 50.0 & \\
\hline \multirow[t]{2}{*}{ Siblings } & I am the only child & 30 & 37.0 & 51 & 63.0 & \multirow[t]{2}{*}{0.181} \\
\hline & I have a sibling & 100 & 45.7 & 119 & 54.3 & \\
\hline
\end{tabular}

\section{Conclusions}

Adolescents constitute a vulnerable population and need careful consideration by mental health care experts and the healthcare system needs to be adapted to provide psychological support despite the lockdown. The COVID - 19 outbreak has resulted in increased depressive symptoms, anger, difficulty in relaxation and sleep disturbances. In addition, the lack of awareness of support and reluctance to use the resources has had a compounding effect on the mental health status of adolescents in India. Adolescents often choose to express their mental health concerns to their parents and siblings. Therefore, it is worth considering family as a potential resource of support for adolescents in this challenging time of need. 


\section{Declarations}

Ethical approval: The study was approved by the Institutional Ethics Committee of Rajarajeswari Medical College, Bangalore

Ethics approval and consent to participate

Consent for publication: Not applicable

Availability of data and materials: The datasets used and/or analyzed during the current study are available from the corresponding author on reasonable request.

Competing interests: The authors declare that they have no competing interests

Funding: None

Authors' contributions:

Brinda Prasanna Kumar: conception and design, acquisition of data, analysis, interpretation of data, drafting the manuscript, revising and final approval.

Adarsh E: design, analysis, interpretation of data, general supervision, guarantor, revising it critically for important intellectual content and final approval of the version to be published

Sahana G: design, analysis, interpretation of data, general supervision, revising it critically for important intellectual content and final approval of the version to be published

Acknowledgements: Not applicable

\section{References}

1. Spear LP. The adolescent brain and age-related behavioral manifestations. Neurosci Biobehav Rev. 2000;24:417-63.

2. Blakemore S-J. The social brain in adolescence. Nat Rev Neurosci. 2008;9:267-77.

3. Casey BJ, Getz S, Galvan A. The adolescent brain. Developmental Review. 2008a;28(1):62-77.

4. Liu X, Kakade M, Fuller CJ, Fan B, Fang Y, Kong J, et al. Depression after exposure to stressful events: lessons learned from the severe acute respiratory syndrome epidemic. Compr Psychiatry. 2012 Jan;53(1):15-23.

5. Sprang G, Silman M. Posttraumatic stress disorder in parents and youth after health-related disasters. Disaster Med Public Health Prep. 2013 Feb;7(1):105-10.

6. Brooks SK, Webster RK, Smith LE, Woodland L, Wessely S, Greenberg N, et al. The psychological impact of quarantine and how to reduce it: rapid review of the evidence. Lancet. 2020 Mar;395(10227):912-20.

7. Dong L, Bouey J. Public Mental Health Crisis during COVID 19 Pandemic China. Emerg Infect Dis. 2020;26:202407.

8. Borner I, Braunstein JW, St Victor R, Pollack J. Evaluation of a 2-question screening tool for detecting depression in adolescents in primary care. Clin Pediatr (Phila). 2010;49(10):947-53. doi:10.1177/0009922810370203.

9. Richardson LP, Rockhill C, Russo JE, et al. Evaluation of the PHQ-2 as a brief screen for detecting major depression among adolescents. Pediatrics. 2010;125(5):e1097-103. doi:10.1542/peds.2009-2712.

10. Clement S, Schauman $\mathrm{O}$, Graham T, et al. What is the impact of mental health-related stigma on help-seeking? A systematic review of quantitative and qualitative studies. Psychol Med. 2015;45(1):11-27. doi:10.1017/S0033291714000129.

11. Maternal newborn, child and adolescent health. WHO; 2017. Cited 2020 August 6 . Available from: https://www.who.int/maternal_child_adolescent/topics/adolescence/mental_health/en/\#: :text=Worldwide\%2010\%2D20\%25\%20 of\%20children,young\%:

12. World Health Organization Coping with stress during the 2019-nCoV outbreak World Health Organization. 2020. Coping with stress during the 2019-nCoV outbreak. https://www.who.int/maternal_child_adolescent/links/covid-19mncah-resources-adolescents-and-youth/en/.

13. Garg K, Kumar CN, Chandra PS. Number of psychiatrists in India: Baby steps forward, but a long way to go. Indian J Psychiatry. 2019;61:104-5.

14. Kumar A. Mental health services in rural India: Challenges and prospects. Health. 2011;3:757-61.

15. National Mental Health Programme. Directorate General of Health Services. Updated 2020 August 27; cited 2020 August 30. Available from: https://dghs.gov.in/content/1350_3_NationalMentalHealthProgramme.aspx.

16. Singh OP. District mental health program-Need to look into strategies in the era of Mental Health Care Act, 2017 and moving beyond Bellary Model. Indian J Psychiatry. 2018;60:163.

17. Nagaraja D. Report of Evaluation of District Mental Health Programme. Bengaluru: NIMHANS; 2003. Cited 2020 August 30 . Available from: https://mhpolicyfileswordpresscom/2011/05/nimhans-report-evaluation-of-dmhppdf.

\section{Figures}




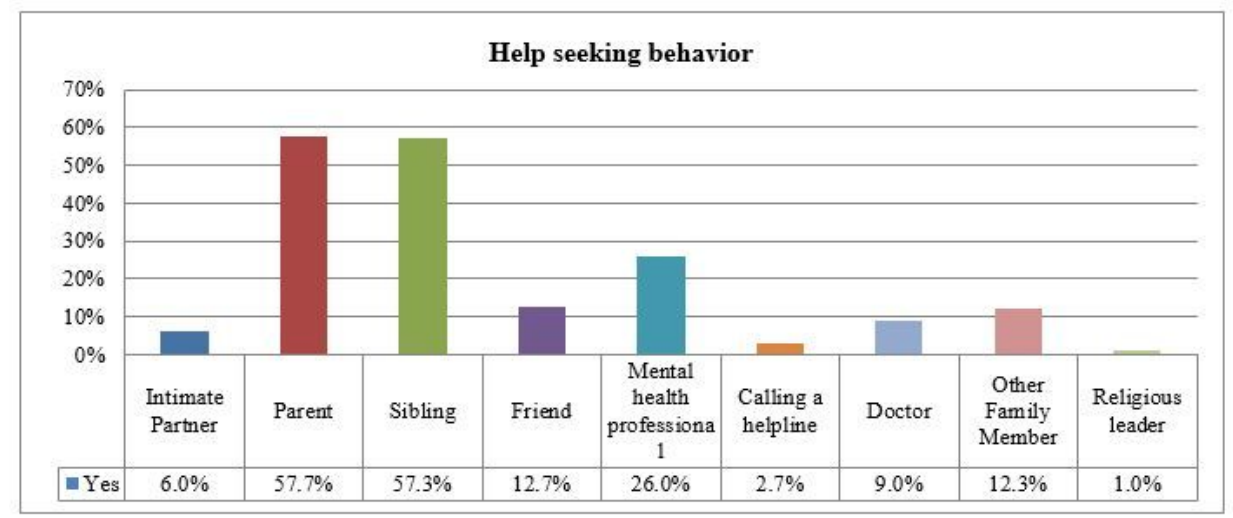

Figure 1

Bar diagram representation of help seeking behavior 